\title{
Do BIM ao CIM: a tecnologia no desenvolvimento de projetos urbanos em Curitiba-PR1
}

\section{From BIM to CIM: the technolgy in the development of urban projects in Curitiba-PR}

\author{
Noguchi, Aline Hissae '; Yamawaki, Yumi2; \\ 1 UTFPR-Curitiba, R. São Marcelino Champagnat, 381 - Maringá-PR, Brasil, \\ alinee.noguchi@gmail.com \\ 2 UTFPR-Curitiba, yumi.yamawaki@gmail.com
}

\begin{abstract}
RESUMO
A utilização do BIM vem se destacando gradativamente na área de construção civil. Atualmente, parcerias de órgãos públicos com setores privados buscam aprimorar os processos para a gestão dos projetos e obras públicas em BIM. Mas, pouco se discute a respeito da Modelagem em Informação da Cidade (CIM) ou de melhorias que visam atender a macroescala. Dessa forma, o trabalho de pesquisa analisou dados teóricos de aplicações internacionais do BIM em direção ao CIM com intuito de enquadrar os conceitos no contexto de projetos urbanos na cidade de Curitiba-PR. Partindo-se de uma referência de aplicação facilitaria a reestruturação para as demais cidades no contexto brasileiro, a fim de promover melhorias na gestão pública, planejamento urbano e monitoramento da cidade. Contudo, o CIM, por ser um assunto inovador e pouco discutido, deverá receber ainda mais o auxílio e incentivo de órgãos públicos, do mesmo aspecto que o BIM vem sendo implementado na capital paranaense.
\end{abstract}

Palavras-chave: CIM, BIM, Gestão pública, Curitiba.

\begin{abstract}
The implementation of BIM on buildings is growing so fast. Nowadays, partnerships between public agencies with private sectors are seeking to improve the process for project management and public buildings in BIM. However, there are a few searches on City Information Modeling (CIM) or improvements aimed at meeting the macro-scale. Thereby, the work searched theoretical data about the international application of BIM and CIM with the purpose of framing the concepts in the context of urban projects in the city of Curitiba-PR. Based on an application reference, it would facilitate the restructuring for other cities in the Brazilian context in order to promote improvements in public management, urban planning and city monitoring. However, because the CIM is an innovative and little discussed, it would be received even more aid and encouragement from public agencies, in the same way that BIM has been implemented in the capital of Paraná.
\end{abstract}

Keywords: CIM, BIM, Public management, Curitiba.

\footnotetext{
${ }^{1}$ NOGUCHI, Aline Hissae; YAMAWAKI, Yumi. Do BIM ao CIM: a tecnologia no desenvolvimento de projetos urbanos em Curitiba-PR. In: II SIMPÓSIO NACIONAL DE GESTÃO E ENGENHARIA URBANA: SINGEURB, 2019, São Paulo. Anais... Porto Alegre: ANTAC, 2019.
} 


\section{INTRODUÇÃO}

A cidade se tornou atrativa pela implementação do BIM em projetos de construção civil por promover edifícios mais eficientes e que possibilitam o desenvolvimento urbano. Com os novos paradigmas, aliado ao crescimento populacional, medidas foram pré-estabelecidas nos países desenvolvidos a fim de garantir melhorias na gestão pública e planejamento urbano.

Atualmente, no Brasil, muitos dados disponibilizados pelos órgãos públicos se encontram desatualizados, os projetos em grande maioria são entregues em CAD (um modelo que exige retrabalho) e as informações não possuem precisão. Dessa forma, a importância do BIM na esfera pública com a inserção do CIM para a melhoria na eficiência da gestão urbana são peças fundamentais para o desenvolvimento de obras público-privadas no país.

\section{OBJETIVO}

Apresentar as experiências da implementação do BIM e do CIM nos países desenvolvidos e contextualizar com base nas práticas desenvolvidas e aplicadas no contexto brasileiro direcionado à cidade de Curitiba-PR.

\section{REVISÃO DE LITERATURA}

\subsection{Do BIM ao CIM}

De acordo com Xun et al. (2013) o CIM (City Information Modeling), seria a integração do BIM com o GIS. A junção destes processos promoveria a obtenção de informações geoespaciais; incluindo os espaços territoriais, referenciando sua latitude e longitude, além de informações detalhadas (Xun et al., 2013).

Logo, a importância do CIM, no cenário urbano, garante tomadas de decisões mais céleres e assertivas com base no modelo de informação da cidade (Almeida e Andrade 2018). Integrar as redes de infra estrutura pública e privada pode oferecer informações precisas e detalhadas. De acordo com Almeida e Andrade (2015), a implementação do CIM na gestão pública promoveria vantagens, como controlar a execução e manutenção das redes instaladas; fundamentar um banco de dados que permita subsidiar de forma mais exata e preventiva revisões de regulamentos urbanos de uso e ocupação do solo e promover tomadas de decisões mais embasadas em situações de crise.

Segundo Sielker e Allmendinger (2018), implementar o CIM não refere-se em proporcionar as cidades em "smart cities", o foco principal é tornar a cidade habitável para os cidadãos baseada em políticas públicas que aproxime o uso de novas tecnologias.

\section{METODOLOGIA}

O trabalho de pesquisa foi desenvolvido a partir de referências bibliográficas a respeito do CIM, nas perspectivas dos autores Xun et al (2013), Almeida e Andrade (2018) e Lima (2016). Com base na fundamentação teórica, buscou-se a aplicação dos conceitos em países internacionais, como Cingapura e Dinamarca, países que vem se destacando em âmbito global em relação às tecnologias em setores públicos-privados.

Ao mesmo tempo, no contexto brasileiro, foi realizado um levantamento de projetos em CIM a fim de realizar um quadro comparativo com as aplicações internacionais. Atualmente, o CIM é um tema pouco discutido e desconhecido por muitos profissionais. Há um baixo índice de pesquisas a respeito e uma porcentagem quase nula de projetos executados. Contudo, o escritório de urbanismo, situado em Curitiba-PR, foi pioneiro em realizar um projeto (piloto) em CIM. Partindo-se deste modelo de projeto, o trabalho de pesquisa buscou informações de aplicações em diferentes escalas e contextos para compreender os inúmeros caminhos que o CIM pode proporcionar, ou até mesmo por qual trajeto o Brasil deve se espelhar. 


\subsection{O contexto internacional}

Primeiramente, a União Europeia teve um passo significativo em relação ao desenvolvimento em BIM no conceito internacional. Pois, possibilitou a criação de uma comissão denominada EUBIM Task Group². Para a comissão, o governo necessita se aproximar da colaboração da implementação da informação digital, por isso o foco principal é desenvolver um manual contendo as considerações técnicas, desenvolvimento cultural e habilidades e benefícios do BIM.

A partir da pesquisa realizada pelos autores Sielker e Allmendinger (2018), foi estipulado uma análise com base nas aplicações do CIM na escala urbana nos principais países de referência à tecnologia BIM. O projeto em análise na Dinamarca, Holstebro Motorway, teve a implementação do BIM com o GIS para diminuir o impacto ambiental por se tratar de uma extensa rodovia que abrange oito cidades locais. Dessa forma, o governo dinamarquês demonstrou como o BIM pode facilitar a reutilização de dados digitais, resolver conflitos durante a fase projetual e construtiva, e desenvolver um modelo exemplar de plataforma (Sielker e Allmendinger, 2018).

Por sua vez, Cingapura está à frente dos demais países. A exigência do uso obrigatório em BIM ocorreu em 2012, e desde o momento, houve o incentivo em desenvolvimento de pesquisas e de recursos financeiros para treinamentos; consultorias; e ações integradas de fomento em BIM (Sielker e Allmendinger, 2018). O projeto de análise escolhido, Virtual Singapore, está em fase de implementação em Cingapura. Trata-se de uma plataforma virtual que disponibiliza dados colaborativos, incluindo mapas, modelo 3D e informações da cidade.

\subsection{O cenário brasileiro: Curitiba-PR}

Semelhante ao EUBIM Task Group, Curitiba inseriu o Plano de fomento BIM com a criação do Laboratório BIM Paraná (LaBIM PR), em 2015. O LaBIM se enquadra em um ambiente de pesquisa e desenvolvimento com o objetivo de definir critérios, formatos, padrões para contratação de projetos e obras públicas em BIM voltado à Secretaria de Infraestrutura e Logística (SEIL).

Ao analisar suas parcerias privadas, verificou-se a existência do projeto (piloto) para a região denominada "Vale do Pinhão". Este Projeto foi desenvolvido e financiado pelo escritório de arquitetura de Curitiba. Segundo Pereira (2019), coordenador do projeto, a ideia de implementação do CIM foi a primeira a ser apresentada para a Prefeitura Municipal de Curitiba, e o primeiro escritório a viabilizar dados para a prefeitura em nível nacional no formato CIM.

Para Lima (2016), a principal dificuldade de implementação do CIM são as delimitações de softwares voltados a esta área. Pereira (2019), por sua vez, utilizou softwares específicos de infraestrutura que contribuísse com uma modelagem tridimensional, georreferenciada e informacional, sem apresentar dificuldades neste quesito. A ideia inicial do projeto seria o aprimoramento para o escritório, simulando dados importantes do Vale do Pinhão, como mapas temáticos quanto as edificações, populações e levantamentos urbanísticos. Como os dados foram relevantes, este projeto foi apresentado para os órgãos públicos municipais.

Afinal, o Vale do Pinhão é considerado uma região atrativa aos olhares do empreendedorismo, por incentivar a implementação de novas tecnologias com intuito de executar a reurbanização e o desenvolvimento ${ }^{3}$. Entretanto, ao apresentar o projeto modelo nos órgãos públicos, a implementação do CIM foi inviável por falta de articulação política (Pereira, 2019). Contudo, as informações e os dados do projeto foram repassados para a

\footnotetext{
2 Informações extraídas pelo site EUBIM Task Group, disponível em: < http://www.eubim.eu/>. Acesso 04 mar.2019.

${ }^{3}$ Informações extraídas pelo site da Prefeitura Municipal de Curitiba, disponível em: < http://valedopinhao.agenciacuritiba.com.br/institucional>. Acesso 15 jun 2019.
} 
prefeitura local, garantindo informações precisas que futuramente poderão ser atribuídas aos profissionais (arquitetos e engenheiros) e mais distante, aos cidadãos.

\section{RESULTADOS}

Com intuito de compreender o progresso do CIM no contexto internacional e a aplicação em Curitiba-PR, foi realizado um quadro comparativo de cada situação. Foi apresentado a aplicação de cada projeto, as metas desenvolvidas, os softwares utilizados e por fim as considerações (quadro 01).

Quadro 1 - Quadro comparativo da implementação do CIM: Países internacionais x Curitiba-PR

\begin{tabular}{|c|c|c|c|}
\hline Análises & Curitiba & Dinamarca & Cingapura \\
\hline Aplicação & Vale do Pinhão & Estrada Holstebro Motorway & $\begin{array}{l}\text { Plataforma Virtual } \\
\text { Singapore }\end{array}$ \\
\hline Abrangência & Projeto pontual & Projeto pontual & Municipal \\
\hline Uso & $\begin{array}{l}\text { Padronização de } \\
\text { projetos municipais }\end{array}$ & $\begin{array}{c}\text { Mensuração de impactos } \\
\text { ambientais }\end{array}$ & $\begin{array}{l}\text { Disponibilização de } \\
\text { dados ao público }\end{array}$ \\
\hline $\begin{array}{l}\text { Público } \\
\text { beneficiado }\end{array}$ & Técnicos municipais & Técnicos municipais & Cidadãos \\
\hline Benefício & Eficiência técnica & Eficiência técnica & Gestão Participativa \\
\hline Fase do BIM & Nível 0 ao 1 & Fase 3 & Fase 4 \\
\hline Metas & $\begin{array}{l}\text { - Desenvolver um } \\
\text { modelo de projeto, e } \\
\text { futuramente } \\
\text { implementar nas demais } \\
\text { regiões da cidade; } \\
\text { - Promover e } \\
\text { disponibilizar os dados } \\
\text { digitais; }\end{array}$ & $\begin{array}{l}\text { - Criar usos consistentes de } \\
\text { dados digitais; } \\
\text {-Reutilizar os dados digitais } \\
\text { durante o ciclo de projeto } \\
\text { e pós-projeto; } \\
\text {-Resolver conflitos durante } \\
\text { as fases de projeto e } \\
\text { construção; }\end{array}$ & $\begin{array}{c}\text { - Colaboração e } \\
\text { tomada de decisão } \\
\text { com base na } \\
\text { integração de dados } \\
\text { digitais; } \\
\text {-Ferramenta de auxílio } \\
\text { para novas pesquisas e } \\
\text { desenvolvimento; } \\
\text { - Promover } \\
\text { acessibilidade para os } \\
\text { cidadãos; } \\
\text { - Melhorias no } \\
\text { planejamento urbano; }\end{array}$ \\
\hline $\begin{array}{l}\text { Sotwares } \\
\text { utilizados }\end{array}$ & $\begin{array}{c}\text { Softwares de } \\
\text { Infraestrutura como: } \\
\text { Infraworks, Civil3D, entre } \\
\text { outros... }\end{array}$ & $\begin{array}{c}\text { Bentley: Microstation, } \\
\text { Navigator, Descartes, } \\
\text { OpenRoads MXROAD e } \\
\text { PowerCivil }\end{array}$ & $\begin{array}{l}\text { Plataforma virtual com } \\
\text { colaboração realizada } \\
\text { pela Dassault Systèmes }\end{array}$ \\
\hline Considerações & $\begin{array}{l}\text { Não houve o incentivo } \\
\text { de outros projetos em } \\
\text { CIM para Curitiba }\end{array}$ & $\begin{array}{c}\text { O governo dinamarquês } \\
\text { pretende promover sua } \\
\text { iniciativa de construção } \\
\text { digital com este projeto, e } \\
\text { introduzir na construção } \\
\text { pública-privada }\end{array}$ & $\begin{array}{c}\text { Ainda não foi } \\
\text { disponibilizado ao } \\
\text { público }\end{array}$ \\
\hline
\end{tabular}

Fonte: Os autores

Com base nestas análises, foi possível chegar nos seguintes resultados conforme a Figura 1:

- Curitiba, por estar em sua fase inicial de aplicação do BIM na esfera pública, ainda 
apresenta as principais dificuldades de inserção: a mudança do CAD para softwares em BIM. Para a capital paranaense chegar na fase de implementação do CIM, os órgãos públicos em conjunto com iniciativas privadas devem passar pelo processo do nível um ao três, de acordo com o gráfico.

- Dinamarca por estar na fase três se direciona ao próximo patamar (Cingapura). O futuro obstáculo seria elaborar uma plataforma virtual com acesso aos dados digitais de forma precisa e rápida.

- Cingapura está à frente dos demais países, sendo considerada um modelo a ser seguido e aplicado na esfera pública e privada. Para Parilusyan, desenvolvedor da Dassault Systèmes, outras cidades usaram o sistema de modelagem 3D para resolver problemas de planejamento urbano, mas a escala de ambição da Virtual Singapore a torna única4.

Figura 1 - Nível do BIM em relação aos Países internacionais x Curitiba-PR

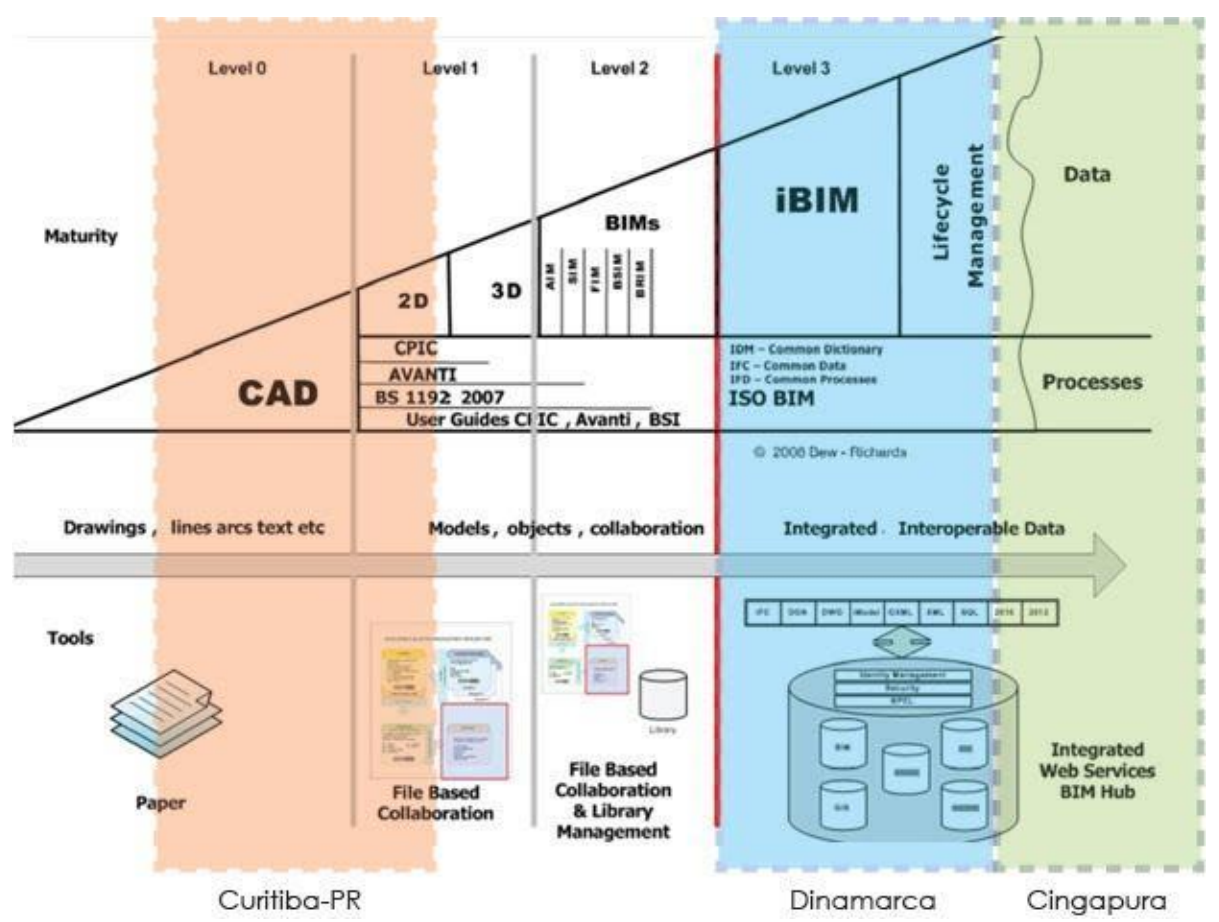

Fonte: Sielker e Allmendinger (2018), adaptado pelos autores (2019)

\section{CONCLUSÃO}

De acordo com a pesquisa apresentada, o CIM é um assunto em fase de amadurecimento, tanto no contexto internacional como nacional.

Curitiba, por estar na fase inicial de implementação em BIM, deve se basear nos países modelos que já passaram por todo o processo da inserção do BIM. Adotando estratégias semelhantes a Dinamarca e Cingapura, por exemplo. É claro que cada país possui sua estratégia distinta, cada caminho escolhido pode apresentar vantagens e desvantagens. Mas, de modo geral, os artifícios utilizados incluem o incentivo do Estado em pesquisas, comitês estratégicos e parcerias privadas.

Já o LaBIM necessita ser difundido no interior do estado paranaense e incentivar a utilização do BIM nos demais municípios. Adotar o BIM na esfera pública seria o passo inicial para um futuro propício à aplicação do CIM. Este tipo de planejamento pode atingir de médios a

\footnotetext{
4 Informações extraídas pelo site:< https://www.reuters.com/article/us-singapore-technology/virtual-singapore-projectcould-be-test-bed-for-planners-and-plotters-idUSKCN1M70U1>. Acesso 13 jun 2019.
} 
longos prazos, por isso, pesquisas devem prosseguir e incentivadas periodicamente, a fim de garantir atualizações tecnológicas e referências de aplicações.

\section{REFERÊNCIAS}

ALMEIDA, F.; ANDRADE, M. A integração entre o BIM e GIS como ferramenta de Gestão Urbana. In: Encontro de Tecnologia de Informação e Comunicação na Construção, 7, 2015, Recife: UFPE, 2015. Anais eletrônico. Disponível em: < https://www.academia.edu/20438337/A_integra\%C3\%A7\%C3\%A3o_entre_BIM_e_GIS_como_ ferramenta_de_gest\%C3\%A3o_urbana>. Acesso em: 09 mar. 2019.

2018. 21-38p.

Considerações sobre o conceito de City Information Modeling. São Paulo: InSitu, 4,

AMORIM, Arivaldo L. Discutindo City Information Modeling (CIM) e conceitos correlatos. Gestão e Tecnologia de Projetos, São Paulo, v.10, n. 2, 87-99p, jul./dez. 2015. Disponível em: <https://doi.org/10.11606/gtp.v10i2.103163>. Acesso em: 09 ma.r 2019.

EUBIM Task Group. 2018. Disponível em: <http://www.eubim.eu/about-the-eu-bim-taskgroup/>. Acesso em: 04 mar. 2019.

GREEN, Erin. 2016. Denmark puts BIM to the test. Disponivel em:

<https://www.engineering.com/BIM/ArticleID/1 1543/Denmark-Puts-BIM-to-the-Test.aspx> Acesso 15 jun 2019.

IEIRI, Ryota. A Singaporean building information modeling Project could revolutionize urban planning. 09 jun. 2016. Disponível em: < https://asia.nikkei.com/Business/Biotechnology/ASingaporean-building-information-modeling-project-could-revolutionize-urban-planning>. Acesso 07 mar. 2019.

LIMA, M. Q. C. Limites e possibilidades do City Information Modeling (CIM) e Planejamento urbano. In: ENANPARQ, 4, 2016, Porto Alegre, 2016. Anais eletrônico. Disponível em: < https://www.anparq.org.br/dvd-enanparq-4/SESSAO\%2014/S14-05-LIMA,\%20M.pdf>. Acesso em: 09 mar. 2019.

PEREIRA, Augusto. Vale do Pinhão. Curitiba, 2019. Arquivo .mp3 (15 minutos). Entrevista pessoal.

SINGAPURE. National Research Foundation. Virtual Singapore. Disponível em: < https://www.nrf.gov.sg/programmes/virtual-singapore>. Acesso: 07 mar. 2019.

ROCHA, Helbert. BIM em obras públicas? - Confira como a plataforma pode ajudar o Brasil, 06 jun. 2017. Disponível em: <https://www.360engenhariabrasil.com/single-post/BuildingInformation-Modeling-no-Brasil>. Acesso em 31 mar. 2019

SIELKER, F.; ALLMENDINGER, P. International experiences: Future Cities and BIM, 2018. University of Cambridge, 1-30p.

XUN, X.; LIEYUN, D.; HANBIN, L.; LING, M. From Building Information Modeling to City Information Modeling. Journal of Information Technology in Construction, 2014. ITcom Vol. 19, p. 292-307. 